\title{
Patterns of long-term survivorship following bevacizumab treatment for recurrent glioma: a case series
}

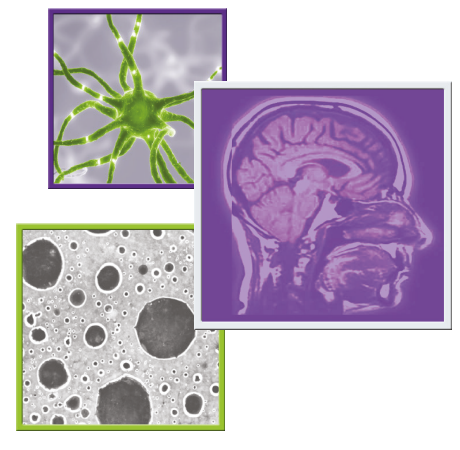

\author{
Liang Yen Liu ${ }^{\ddagger}, 1$, Matthew S Ji ${ }^{\ddagger 1}$, Nhung T Nguyen ${ }^{\ddagger 1}$, Frances E Chow ${ }^{1}$, Donna M Molaie ${ }^{1}$, \\ Sean T Pianka1, Richard M Green², Linda M Liau³, Benjamin M Ellingson ${ }^{4}$, Phioanh L \\ Nghiemphu', Timothy F Cloughesy ${ }^{1}$ \& Albert Lai*,1 iD \\ ${ }^{1}$ Department of Neurology, University of California, Los Angeles, 710 Westwood Plaza RNRC \#1-230, Los Angeles, CA 90095, USA \\ ${ }^{2}$ Department of Neurology, Kaiser Permanente, Southern California, 4867 W Sunset Blvd, Los Angeles, CA 90027, USA \\ ${ }^{3}$ Department of Neurosurgery, University of California, Los Angeles, Edie \& Lew Wasserman Building, 300 Stein Plaza, Ste. 420, \\ Los Angeles, CA 90095, USA \\ ${ }^{4}$ Department of Radiological Sciences \& Psychiatry, University of California, Los Angeles, 924 Westwood Blvd, Ste. 615, Los \\ Angeles, CA 90024, USA \\ *Author for correspondence: Tel.: +1 310825 5321; Fax: +1 310825 0644; albertlai@mednet.ucla.edu \\ ¥ Authors contributed equally
}

\begin{abstract}
Aim: Long-term survivors (LTS) after glioma recurrence while on bevacizumab (Bev) therapy are rarely reported in the current literature. The purpose of this case series is to confirm the existence of and describe a large cohort of recurrent glioma LTS treated with Bev (Bev-LTS). Patients \& methods: We identified Bev-LTS as patients with post-Bev initiation survival times of $\geq 3$ years among 1397 Bev treated recurrent glioma patients. Results: Among 962 grade-IV, 221 grade III, and 214 grade II Bev-treated glioma patients, we identified $28(2.9 \%), 14(6.3 \%)$ and $8(3.7 \%)$ Bev-LTS patients, respectively. 45 Bev-LTS patients recurred on Bev, with 36 of those patients continuing therapy. Conclusion: Our study shows that a small portion of grade-IV, -III, and -II glioma patients can have long-term survival on Bev therapy even after Bev recurrence.
\end{abstract}

First draft submitted: 2 April 2019; Accepted for publication: 15 May 2019; Published online: 11 July 2019

Keywords: bevacizumab • glioblastoma $\bullet$ glioma $\bullet$ long term $\bullet$ progression $\bullet$ recurrence $\bullet$ relapse $\bullet$ survivor

Gliomas are the most common primary brain cancers in adults and are incurable for most patients due to their highly infiltrative nature. Despite treatment with standard-of-care therapy, almost all patients relapse. Treatment options at recurrence are limited, and patients often experience poor prognoses with neurological disability and worsened quality of life. Anti-angiogenic therapy with bevacizumab (Bev), a monoclonal antibody targeting VEGF [1,2], is commonly used for the treatment of recurrent glioblastoma (GBM) and recurrent high-grade gliomas since receiving expedited approval from the US FDA in 2009 and full approval for recurrent GBM in 2017.

While Bev treatment at recurrence has improved management of symptoms and progression-free survival (PFS), there has been no prospective data showing improvement in overall survival (OS), and Bev is associated with many potential side effects [3-5]. The randomized Phase III EORTC 26101 trial showed that combination therapy of Bev and lomustine resulted in an OS and PFS of 9.1 and 4.2 months, respectively [4]. Similarly, three Phase II studies for recurrent GBM patients treated with Bev [6-8] and a retrospective study on deferred Bev use for recurrent GBM patients [9] reported median OS and PFS ranges of 8-9.2 and 3-4.2 months, respectively. In another recurrent GBM study, Iwamoto et al. reported a median OS of only 4.5 months after tumor recurrence on Bev therapy [10]. Bev therapy has not demonstrated efficacy in the treatment of recurrent grade II and -III glioma patients either. The Phase II EORTC TAVAREC trial, which is currently the largest Bev therapy trial that has been done for recurrent grade II and III non co-deleted 1p19q glioma patients, showed that combination therapy of temozolomide and Bev did not significantly improve OS and PFS compared with temozolomide monotherapy [11].

Currently, 5-year overall survival rates are 5.5\% for GBM, 29.7-49.7\% for grade II and -III astrocytomas, and $56.7-80.9 \%$ for grade II and -III oligodendrogliomas [12]. However, these numbers do not necessarily take

Future Medicine 


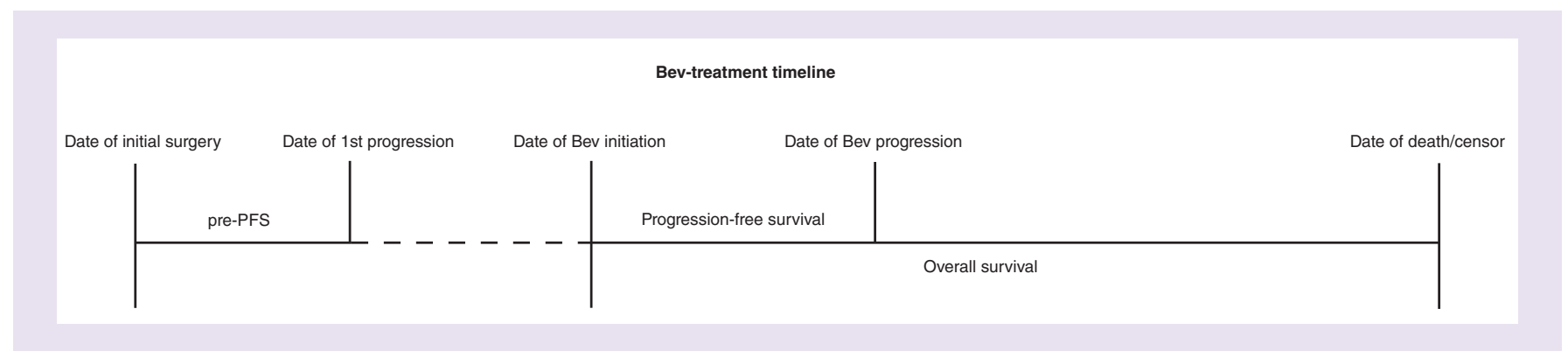

Figure 1. pre-PFS, time between date of diagnosis and date of the first recurrence; progression-free survival, time between date of bevacizumab (Bev) initiation and date of first tumor recurrence after bevacizumab initiation; overall survival, time between date of bevacizumab initiation and date of death or censor.

Pre-PFS: Pre progression-free survival.

recurrence into account since many survivors do not have recurrence. There are few reports of long-term surviving recurrent high- and low-grade glioma patients who have had prolonged survival [13-17], but these studies do not specifically focus on these long-term surviving patients, especially in the context of Bev. For recurrent GBM patients treated with Bev, two case reports each described a patient achieving tumor-free status at 26 months and 3 years, respectively, with the latter patient receiving surgical re-resection and irinotecan in conjunction with Bev [18,19]. A retrospective analysis of Bev treatment in a cohort of 16 recurrent GBM patients showed a median post-progression survival of 39.6 months [20]. However, to our knowledge, there are currently few studies in the literature on Bevtreated long-term survivors among recurrent grade II and -III glioma patients. Therefore, the goal of this case series is to present and describe the characteristics of a large cohort of long-term survivors among recurrent grade-IV, -III, and -II glioma patients who were treated with Bev.

\section{Methods}

LTS cohort selection \& survival interval definitions

This study utilized an electronic database query of recurrent adult glioma patients treated with Bev who were seen at University of California, Los Angeles (UCLA) and Kaiser Permanente, Los Angeles (KPLA) from 2004 to 2017. From this database, we identified 1397 patients who were initially diagnosed with grade-IV (962), -III (221), and -II (214) gliomas and treated with Bev at recurrence. Informed consent for this University of California Los Angeles/Kaiser Permanente Los Angeles Institutional Review Board-approved retrospective database study was obtained from all patients. From these initial 1397 patients, Bev-LTS (long-term survivors after glioma recurrence while on Bev therapy) patients were identified as those who had an overall survival (OS) of $\geq 3$ years between the date of Bev initiation and the date of death or censor (Figure 1). Since there is no formal clinical definition of long-term survival after Bev initiation, we used an OS of $\geq 3$ years as our criteria for Bev-LTS. Pre progression-free survival (pre-PFS) was defined as the time between the date of diagnosis and date of the first recurrence (Figure 1). Progression-free survival was defined as the time between the date of recurrence when Bev was initiated and the date of subsequent tumor recurrence (Figure 1). Tumor recurrence was determined by the treating clinician according to the response assessment in neuro-oncology (RANO) criteria. Indeterminate (pseudo) progressors were identified as patients who had their first recurrence within 3 months of radiation therapy. These patients were still included in this study as we believed that it would be important to report on any long-term surviving recurrent glioma patient. However, they were excluded when calculating the median values for OS, pre-PFS and PFS for the rest of the cohort. Median OS, pre-PFS and PFS were calculated using Kaplan-Meier analysis.

\section{Patient characteristics}

Age at diagnosis and age at Bev initiation were defined as the patient age at the date of initial surgery and at the date of the first Bev treatment, respectively. All patients within the Bev-LTS cohort had histologically confirmed tumor pathology of glioma, which ranged from WHO grade II to -IV. All diagnoses (grade) are initial diagnoses even if malignant transformation was later suspected by imaging or histologically confirmed. Patients who were diagnosed prior to 2016 were not adjusted to the 2016 WHO glioma classification system [21]. Extent of resection (EOR) at initial tumor diagnosis was defined as gross total resection, subtotal resection or biopsy. Subtotal resection was 
defined as a resection that removed $<90 \%$ of the gross tumor mass. Karnofsky Performance Status (KPS) scores at Bev initiation were obtained. Patient progression after Bev initiation was also recorded. Molecular biomarker information in terms of $I D H 1$ and $I D H 2$ mutation statuses and $M G M T$ promoter methylation status, were collected when available.

\section{Tumor measurements}

The 2D tumor sizes were measured for only the grade-IV glioma (GBM) patients at the date of tumor recurrence when Bev treatment was initiated using axial postcontrast T1-weighted MRI scans. These measurements were independently obtained by two separate investigators to ensure inter-rater reliability. To obtain the 2D tumor size, the maximum diameter length of the contrast enhancing regions in each MRI scan and the length of the perpendicular axis were multiplied together. In the context of multifocal tumors, each part of the tumor was measured as described previously before adding the separate measurements together to obtain the total tumor size. Although the conventional 2D measurement technique has been shown to not be as predictive of OS relative to the 3D measurement technique [22], it still has been shown to associate with OS in recurrent GBM patients who were treated with Bev [23].

\section{Stratification of LTS cohort}

We separated the GBM Bev-LTS cohort into five subgroups to ascertain long-term post-Bev survival patterns: group A (early Bev initiation and long-term PFS), group B (late Bev initiation and long-term PFS), group C (late Bev initiation and short-term PFS), group D (indeterminate [pseudo] progressors) and group E (early Bev initiation and short-term PFS). Bev initiation during first recurrence was defined as 'early Bev initiation', while Bev initiation during later recurrences (second and up) was defined as 'late Bev initiation'. Piccioni et al. found that the median PFS for recurrent GBM patients was 4.1 months, similar to median PFS values found from other studies [6-10]. Therefore, a PFS $\geq 4.1$ months was defined as 'long-term PFS', while a PFS $<4.1$ months was defined as 'short-term PFS'. Median tumor measurements, PFS and OS were only calculated for grade-IV patients within these groups. Since there were only a small number of low-grade glioma patients, these patients were added in these groups according to the same GBM-derived PFS threshold.

\section{Description of cases}

Overall general and individual patient characteristics and data are summarized in Table 1 and Supplementary Table 1, respectively. From the initial cohort of 1397, 50 were identified as Bev-LTS patients. Out of all diagnosed grade-IV, -III, and -II glioma patients, 28 (2.9\%), 14 (6.3\%), and 8 (3.7\%) were identified as Bev-LTS, respectively. 27 were initially diagnosed with grade IV GBM and one with grade IV gliosarcoma (GLIO). Six were diagnosed with grade III anaplastic astrocytoma (AA), three with grade III anaplastic mixed gliomas (AMG) and five with grade III anaplastic oligodendrogliomas (AO). Two were diagnosed with grade II low-grade astrocytoma (LA), one with grade II low-grade mixed glioma (LGMO), and five with grade II low-grade oligodendrogliomas (LO). Three of the $27 \mathrm{GBM}, 1$ of the $6 \mathrm{AA}$ and the $2 \mathrm{LA}$ patients were identified as indeterminate (pseudo) progressors.

Information on extent of tumor resection, KPS, incidence of recurrence on Bev treatment and tumor size measurement is also summarized (Table $1 \&$ Supplementary Table 1). Two GBM patients had additional tumor resections with unknown extent of resection. One patient had an additional tumor resection 25 months before Bev initiation and the other patient had two additional resections, with the last resection being 60 months before Bev initiation. One AA patient had an additional gross tumor resection 6 months before Bev initiation with no change in pathology. No patients had repeat resection prior to Bev to establish possible increase in tumor grade. Nearly all patients $(\mathrm{n}=40)$ with known KPS data at Bev initiation had a KPS $\geq 70$. Only one GBM and one LO patient had a KPS $<70$. Progression after Bev initiation was common place among the cohort, with 43 out of the 50 patients having tumor recurrence while on Bev. Six grade IV patients, one of them being an indeterminate progressor, and one AA indeterminate (pseudo) progressor were the only patients who did not progress on Bev. Despite continued progression, most patients continued Bev therapy, with only five GBM patients and one LO patient discontinuing Bev. For tumor size measurements, excluding the three GBM indeterminate (pseudo) progressor patients, the median 2D tumor size for the grade IV patients was $1306 \mathrm{~mm}^{2}$.

Biomarker data on $I D H 1, I D H 2$ and $M G M T$-methylation status at initial diagnosis were limited and are summarized generally and individually (Table $1 \&$ Supplementary Table 1). Ten GBM patients were $I D H 1$ wild-type while another two were IDH1 mutant. There were eight known IDH2 mutation statuses known for five GBM, two 


\section{Table 1. Summary of patient demographics.}

\begin{tabular}{|c|c|c|c|c|c|}
\hline \multicolumn{6}{|c|}{ Summary of patient demographics $(\mathrm{N}=50)$} \\
\hline Initial diagnosis & Grade IV $(n=28)$ & $\begin{array}{l}\text { Grade III } \\
\text { astrocytomas/mixed } \\
\text { gliomas }(n=9)\end{array}$ & $\begin{array}{l}\text { Grade III } \\
\text { oligodendroglioma }(n=5)\end{array}$ & $\begin{array}{l}\text { Grade II } \\
\text { astrocytoma/mixed } \\
\text { glioma }(n=3)\end{array}$ & $\begin{array}{l}\text { Grade II oligodendroglioma } \\
(n=5)\end{array}$ \\
\hline $\begin{array}{l}\text { Age at bevacizumab } \\
\text { initiation (years) median } \\
\text { (range) }\end{array}$ & $56.3(23.8-68.6)$ & $49.7(21.7-60)$ & $40.2(39.5-65)$ & $44(42.9-45.2)$ & $45.2(34-58)$ \\
\hline - Male (\%) & $17(61 \%)$ & $6(67 \%)$ & $4(80 \%)$ & $2(67 \%)$ & $3(60 \%)$ \\
\hline - Female (\%) & $11(39 \%)$ & $3(33 \%)$ & $1(20 \%)$ & $1(33 \%)$ & $2(40 \%)$ \\
\hline \multicolumn{6}{|l|}{ KPS: } \\
\hline$-\geq 70(\%)$ & $23(82 \%)$ & $6(67 \%)$ & $4(80 \%)$ & $2(67 \%)$ & $3(60 \%)$ \\
\hline$-<70(\%)$ & $1(4 \%)$ & $0(0 \%)$ & $0(0 \%)$ & $0(0 \%)$ & $1(20 \%)$ \\
\hline - IDH1 wild-type (\%) & $10(36 \%)$ & $1(11.1 \%)$ & $0(0 \%)$ & $0(0 \%)$ & $0(0 \%)$ \\
\hline - Unknown (\%) & $16(57 \%)$ & $4(44.4 \%)$ & $4(80 \%)$ & $3(100 \%)$ & $5(100 \%)$ \\
\hline \multicolumn{6}{|l|}{ IDH2 mutation status: } \\
\hline - IDH2 mutated (\%) & $0(0 \%)$ & $0(0 \%)$ & $0(0 \%)$ & $0(0 \%)$ & $0(0 \%)$ \\
\hline - IDH2 wild-type (\%) & $5(18 \%)$ & $3(33 \%)$ & $0(0 \%)$ & $0(0 \%)$ & $0(0 \%)$ \\
\hline - Unknown (\%) & $23(82 \%)$ & $6(67 \%)$ & $5(100 \%)$ & $3(100 \%)$ & $5(100 \%)$ \\
\hline \multicolumn{6}{|l|}{ MGMT-methylation status: } \\
\hline - MGMT methylated (\%) & $4(14 \%)$ & $2(22 \%)$ & $1(20 \%)$ & $0(0 \%)$ & $0(0 \%)$ \\
\hline $\begin{array}{l}\text { - MGMT unmethylated } \\
(\%)\end{array}$ & $3(11 \%)$ & $1(11 \%)$ & $0(0 \%)$ & $1(25 \%)$ & $0(0 \%)$ \\
\hline - Unknown (\%) & $21(75 \%)$ & $6(67 \%)$ & $4(80 \%)$ & $3(75 \%)$ & $5(100 \%)$ \\
\hline - Radiation (\%) & $27(96 \%)$ & $8(100 \%)$ & $3(60 \%)$ & $3(75 \%)$ & $3(60 \%)$ \\
\hline - Temozolomide (\%) & $25(89 \%)$ & $7(88 \%)$ & $4(80 \%)$ & $3(75 \%)$ & $1(20 \%)$ \\
\hline - Additional surgery (\%) & $2(7 \%)$ & $1(12 \%)$ & $0(0 \%)$ & $0(0 \%)$ & $0(0 \%)$ \\
\hline \multicolumn{6}{|c|}{ Concurrent treatments with bevacizumab: } \\
\hline - Irinotecan (\%) & $13(46 \%)$ & $6(67 \%)$ & $3(60 \%)$ & $3(100 \%)$ & $4(80 \%)$ \\
\hline - Lomustine (\%) & $12(43 \%)$ & $5(56 \%)$ & $1(20 \%)$ & $1(33 \%)$ & $2(40 \%)$ \\
\hline - Carboplatin (\%) & $8(28 \%)$ & $6(67 \%)$ & $2(40 \%)$ & $2(66 \%)$ & $3(60 \%)$ \\
\hline - Etoposide (\%) & $4(14 \%)$ & $5(56 \%)$ & $3(60 \%)$ & $1(33 \%)$ & $1(20 \%)$ \\
\hline - Temozolomide (\%) & $4(14 \%)$ & $2(22 \%)$ & $1(20 \%)$ & $2(67 \%)$ & $1(20 \%)$ \\
\hline - Radiation (\%) & $7(25 \%)$ & $2(22 \%)$ & $1(20 \%)$ & $1(33 \%)$ & $0(0 \%)$ \\
\hline - Craniotomy (\%) & $2(7 \%)$ & $0(0 \%)$ & $0(0 \%)$ & $1(33 \%)$ & $0(0 \%)$ \\
\hline - Procarbazine (\%) & $1(3 \%)$ & $1(11 \%)$ & $1(20 \%)$ & $0(0 \%)$ & $0(0 \%)$ \\
\hline \multicolumn{6}{|c|}{ Recurrence when bevacizumab was initiated: } \\
\hline - First recurrence (\%) & $19(68 \%)$ & $6(67 \%)$ & $0(0 \%)$ & $3(100 \%)$ & $1(20 \%)$ \\
\hline - Later recurrences $(\%)$ & $9(32 \%)$ & $3(33 \%)$ & $5(100 \%)$ & $0(0 \%)$ & $4(80 \%)$ \\
\hline $\begin{array}{l}\text { Recurrence on } \\
\text { bevacizumab treatment } \\
\text { (\%) }\end{array}$ & $22(79 \%)$ & $8(89 \%)$ & $5(100 \%)$ & $3(100 \%)$ & $5(100 \%)$ \\
\hline
\end{tabular}




\begin{tabular}{|c|c|c|c|c|c|}
\hline \multicolumn{6}{|c|}{ Summary of patient demographics $(\mathrm{N}=50)$} \\
\hline Initial diagnosis & Grade IV $(n=28)$ & $\begin{array}{l}\text { Grade III } \\
\text { astrocytomas/mixed } \\
\text { gliomas }(n=9)\end{array}$ & $\begin{array}{l}\text { Grade III } \\
\text { oligodendroglioma }(n=5)\end{array}$ & $\begin{array}{l}\text { Grade II } \\
\text { astrocytoma/mixed } \\
\text { glioma }(n=3)\end{array}$ & $\begin{array}{l}\text { Grade II oligodendroglioma } \\
(n=5)\end{array}$ \\
\hline $\begin{array}{l}\text { Continuation of } \\
\text { treatment after tumor } \\
\text { recurrence on } \\
\text { bevacizumab (\% of } \\
\text { patients who recurred on } \\
\text { bevacizumab) }\end{array}$ & $17(77 \%)$ & $8(100 \%)$ & $4(80 \%)$ & $3(100 \%)$ & $4(100 \%)$ \\
\hline $\begin{array}{l}\text { 2D tumor measurements } \\
\left(\mathrm{mm}^{2}\right) \text { median }\end{array}$ & 1306 & NA & NA & NA & NA \\
\hline
\end{tabular}

KPS: Karnofsky Performance Status score at bevacizumab initiation; NA: Not applicable.

\section{Table 2. Summary of survival patterns.}

\begin{tabular}{|c|c|c|c|c|c|}
\hline \multicolumn{6}{|c|}{ Summary of survival patterns $(\mathrm{N}=44)$} \\
\hline Initial diagnosis & Grade-IV $(n=25)$ & $\begin{array}{l}\text { Grade III } \\
\text { astrocytomas/mixed } \\
\text { gliomas }(n=8)\end{array}$ & $\begin{array}{l}\text { Grade III } \\
\text { oligodendroglioma }(n=5)\end{array}$ & $\begin{array}{l}\text { Grade II } \\
\text { astrocytomas/mixed } \\
\text { gliomas }(n=1)\end{array}$ & $\begin{array}{l}\text { Grade II oligodendroglioma } \\
(n=5)\end{array}$ \\
\hline $\begin{array}{l}\text { Pre-PFS (months) median } \\
(95 \% \mathrm{Cl})\end{array}$ & $8.9(7.6-13.0)$ & $18.0(11.4-\mathrm{NR})$ & 29.8 (12.8-NR) & NA & 24.5 (14.8-NR) \\
\hline $\begin{array}{l}\text { PFS (months) median } \\
(95 \% \mathrm{Cl})\end{array}$ & $28.9(15.2-36.1)$ & 7.1 (5.2-NR) & 22.2 (13.4-NR) & NA & 38.8 (18.0-NR) \\
\hline $\begin{array}{l}\text { OS (months) median } \\
(95 \% \mathrm{Cl})\end{array}$ & $52.8(44.5-64.4)$ & 50.5 (47.6-NR) & 52.6 (51.3-NR) & NA & $109.8(81.0-N R)$ \\
\hline \multicolumn{6}{|c|}{$\begin{array}{l}\text { The pre-PFS, PFS and OS for grade II astrocytomas/mixed gliomas were not calculated since there was only one patient (one low grade mixed glioma) who was not considered an } \\
\text { indeterminate (pseudo) progressor, who were defined as patients who had initial tumor size increase within } 3 \text { months of radiation therapy. Three such grade-IV and one such grade III } \\
\text { astrocytoma/mixed glioma patients were excluded as well. } \\
\text { CI: } \\
\text { Confidence interval; NA: Not applicable; NR: Upper limit of } 95 \% \text { confidence interval not reached; OS: Overall survival, time between the date of bevacizumab initiation to date of death } \\
\text { or censor; PFS: Progression-free survival, time between date of bevacizumab initiation and date of the first recurrence after bevacizumab initiation; pre-PFS: Pre progression-free survival, } \\
\text { time between date of diagnosis and date of the first recurrence. }\end{array}$} \\
\hline
\end{tabular}

AA and one AMG patients, all of whom were $I D H 2$ wild-type. Among the four known $M G M T$-methylated GBM patients, two were $I D H 1$ wild-type and two were $I D H 1$ mutant. Among the three known MGMT-unmethylated GBM patients, all were $I D H 1$ wild-type. Most patients also concurrently received additional treatments during Bev treatment, including chemotherapy agents such as irinotecan, carboplatin, lomustine, etoposide, and procarbazine, as well as further radiation and surgical treatments (Table $1 \&$ Supplementary Table 1).

Patient survival patterns, excluding the survival data from the indeterminate (pseudo) progressors, are summarized in Table 2. The median pre-PFS values of the grade IV group and the combined grade III AA/AMG group were 8.9 and 18 months, respectively. The AO and LO groups had the highest median pre-PFS values, 29.8 and 24.5 months, respectively. The median PFS of the LO group was 38.8 months, which was the highest of all groups. The grade IV and the AO groups had the next highest PFS medians of 28.9 and 22.2 months, respectively, while the AA/AMG group had the lowest median PFS of 8 months. The median OS values of the grade IV, AO and AA/AMG groups were all similarly around 51 months. The LO group had the highest median OS of 109.8 months. The longest surviving patient within our cohort was an AO patient who had an OS $>12$ years. Within the AA group, there was one patient with an OS $>10$ years. Within the GBM group, there were two patients with an OS $>10$ years and an additional eight patients with an OS $>5$ years. Furthermore, there was one AMG patient and an additional one $\mathrm{AO}$ patient with an OS $>5$ years. There was only one patient within the grade II LA/LGMO group who was not considered an indeterminate (pseudo) progressor. This patient had a pre-PFS, PFS and OS of 6.4, 24.5 and 43.3 months, respectively. Among the indeterminate (pseudo) progressors, there were one GBM and one LA patients with an OS $>5$ years.

Overall general patient characteristics and data among the five subgroups are summarized in Table 3. There were no Bev-LTS patients that received Bev initiation at early recurrence and had a short-term PFS (group E). Therefore, this group was not represented in Table 3. The distribution of the PFS values of these patients, which was used to separate patients into the remaining four subgroups, was summarized in Supplementary Figure 1. Group A ( $\mathrm{n}=23)$ 
Table 3. Summary of patient characteristics among subgroups defined by early-late recurrence/short-long progression-free survival.

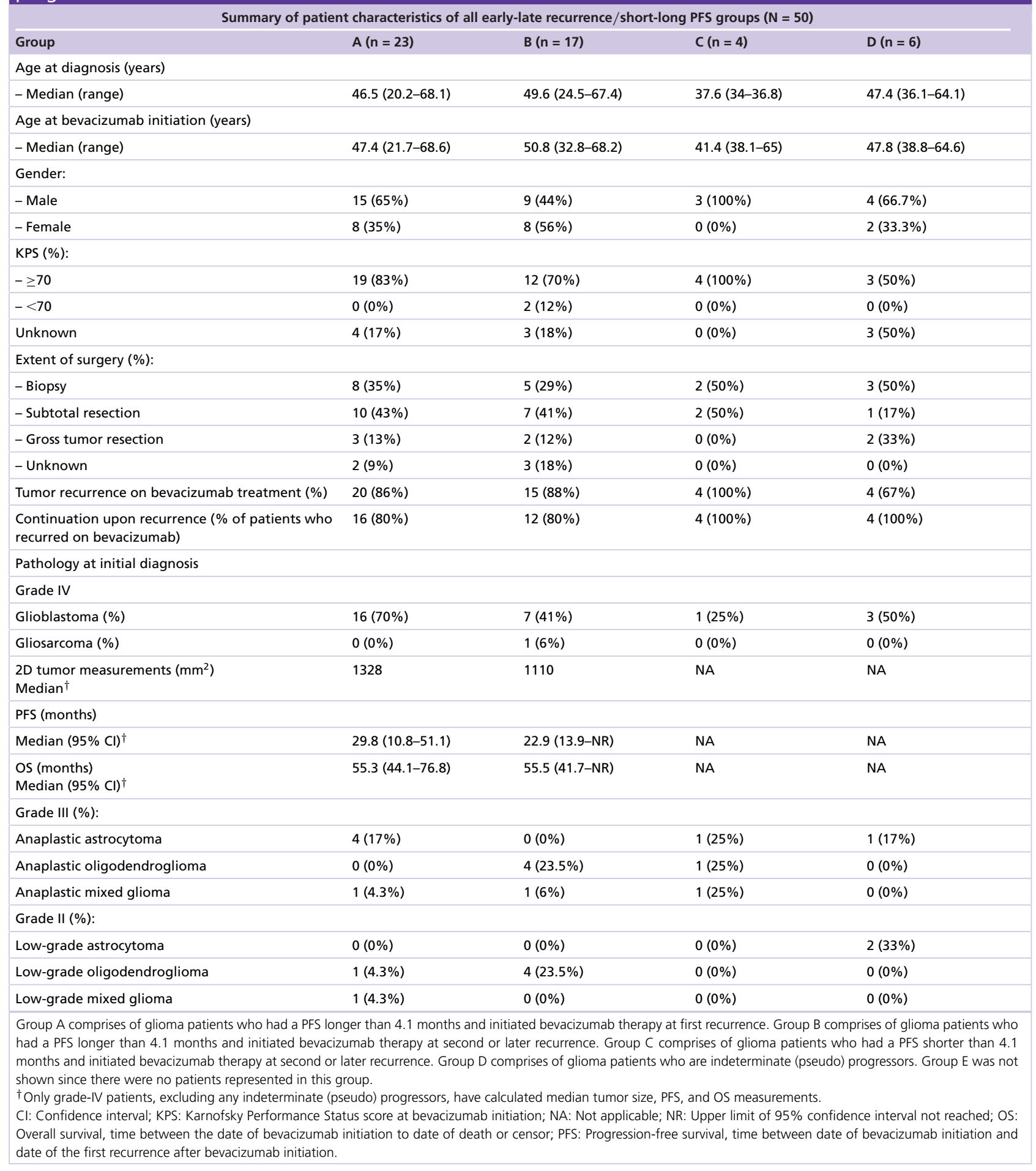

contained patients with grade IV GBM $(n=16)$, grade III AA $(n=4)$ and AO $(n=1)$, and grade II LO $(n=1)$ and LGMO $(n=1)$. For the 16 grade IV patients in Group A, the median PFS and OS were 29.8 and 55.3 months, respectively. Group B contained patients with grade IV GBM $(n=7)$, grade IV GLIO $(n=1)$, grade III AO $(\mathrm{n}=4)$, and grade III AMG $(\mathrm{n}=1)$, while Group C contained patients with grade IV GBM $(\mathrm{n}=1)$, grade III AA $(\mathrm{n}=1)$, grade III AO $(\mathrm{n}=1)$, and grade III AMG $(\mathrm{n}=1)$. Group D contained indeterminate (pseudo) progressors 
with grade IV GBM $(\mathrm{n}=3)$, grade III AA $(\mathrm{n}=1)$, and grade II LA $(\mathrm{n}=1)$. Group B's grade IV patients had median PFS and OS values of 22.9 and 55.5 months, respectively. The median tumor sizes among Group A's and B's grade IV patients were 1328 and $1110 \mathrm{~mm}^{2}$, respectively.

\section{Discussion}

Long-term survival in recurrent glioma patients treated with Bev is rarely reported in the current literature, especially for grade II and III glioma patients. The purpose of this study is to present these rare patients and describe their characteristics. Among the initial cohort of 1397 patients treated with Bev at recurrence, there were 962 grade IV, 221 grade III and 214 grade II glioma patients, but only 28 grade IV, 14 grade III and 8 grade II Bev-LTS patients were identified. This means that only $2.9 \%$ of grade IV, $6.3 \%$ of grade III and $3.7 \%$ of grade II patients survived 3 years or longer after initiating Bev. These findings corroborate with the fact that the majority of glioma patients who experience recurrence continue to have very poor prognoses on Bev therapy. Reported median OS values for recurrent grade-IV and -II/III patients treated with Bev are approximately 9 and 13 months, respectively [4,11]. However, many patients in our cohort were able to survive for over 5 years and up to over 12 years. This may indicate that there are some glioma patients treated with Bev who may survive much longer than expected.

We noticed that the grade II nonoligodendroglioma patients had unusually low pre-PFS values. This could be explained by the fact that both the LA patients were considered indeterminate (pseudo) progressors (patients with tumor recurrence within 3 months of radiation therapy) while the LGMO patient had a pre-PFS of 6.4 months. This LGMO patient did have aggressive features noted on the pathology report, which cautioned that there might be sampling error as the initial surgery was only a biopsy.

Nearly all our Bev-LTS experienced tumor recurrence on Bev therapy and survived 3 years or longer, despite the fact that recurrent glioma patients who experience tumor recurrence on Bev therapy typically have very poor outcomes [10,14]. Interestingly, 36 of these patients who initially experienced tumor recurrence on Bev therapy still experienced long-term survival on continued Bev treatment. Furthermore, in our observations of the patient clinical histories, we saw that many patients had repeated subsequent recurrences on Bev-containing regimens. Bev is currently one of the most utilized therapies available for the treatment of recurrent gliomas, and the utilization of Bev in a repeated fashion despite repeated recurrences is common clinical practice [24]. Indeed, the contribution of Bev to long-term survival in our patient cohort is unclear since many patients were also on common concurrent therapies, such as irinotecan and carboplatin (Table 1). More importantly, clinicians should be aware of the existence, albeit rare, of long-term surviving patients despite recurrence on Bev.

The tumor size median for grade IV glioma Bev-LTS patients was $1306 \mathrm{~mm}^{2}$. Nguyen et al. reported a median 2D tumor size of $1196 \mathrm{~mm}^{2}$ for a cohort of recurrent GBM patients treated with Bev, and showed that patients with larger pre-Bev tumor burdens received the most benefit from Bev [23]. Thus, relative to this threshold of $1196 \mathrm{~mm}^{2}$, the tumor size medians for the grade IV Bev-LTS cohort would be considered large.

We found that KPS at the time of Bev initiation was $\geq 70$ for nearly all the Bev-LTS of our cohort. KPS has consistently been identified in the current literature as a potential predictor for better PFS and OS for recurrent GBM patients treated with Bev [25-27]. Therefore, a good functional status may be required to have a chance to experience long-term survival with Bev treatment.

The stratification of our cohort into group showed that our patients fell mostly in either group A or B. When comparing the survival patterns of the grade IV patients within these two groups, the median OS and PFS were similar (Table 3). This indicates that deferring Bev treatment until later recurrences appears not to be detrimental to the OS as reported previously [9,28]. There were only three patients in Group C and none in Group E, which suggests that long-term surviving patients with shorter PFS values after Bev initiation (Groups $\mathrm{C}$ and $\mathrm{E}$ ) may not be as frequent as those with longer PFS values (Groups A and B). Bev may also lead to more aggressive patterns of tumor growth in later recurrences after recurrence on Bev treatment $[10,29]$, which raises the possibility that Bev-LTS patient tumors do not undergo these changes. It is not clear if there is an optimal time to stop Bev, especially with the possibility that a small subset can experience long-term survival.

This study was limited for various reasons. The definition of Bev-LTS is not formally defined and was based only on survival times from previous clinical studies of GBM patients $[4,6-9,11]$. Additionally, the reported frequencies of Bev-LTS patients must be considered estimates due to the likelihood of inherent selection biases within our patient group. As a retrospective descriptive study, this study lacked any comparison of control groups and consistent concomitant treatments per recurrence. There were glioma patients within our cohort who survived a significant amount of time before starting Bev, which may suggest these patients could have had long-term survival irrespective 
of Bev treatment. This study does not provide any mechanistic explanations for what underlying genetic factors may cause Bev-LTS to have favorable responses to Bev, especially since biomarker data were limited. There are some studies that suggest certain genetic alterations, such as MGMT-methylation and ATRX alteration, and PDGFRA alteration, are valuable prognostic factors for long-term survival in GBM patients [30,31]. Therefore, additional studies with more complete patient genetic biomarker information could be valuable in developing criteria for identifying potential Bev-LTS. Lastly, the pathological diagnosis has not been uniformly made using 2016 WHO guidelines, since many of the patients were diagnosed prior to 2016 and lack necessary biomarker data.

\section{Conclusion}

In summary, we have identified a group of recurrent grade-IV, -III, and -II glioma patients exhibiting long-term survival on Bev treatment. As a descriptive study, we cannot draw conclusions about whether Bev is responsible for LTS in those patients or how such LTS patients might be identified propsectively. As such, the pervasive clinical use of Bev for many different recurrent gliomas coupled with the fact that there are some glioma patients receiving Bev therapy who can survive significantly longer than the norm warrant further studies that can assess other potential clinical and molecular predictive factors for Bev-LTS and determine optimal timing and utilization of Bev for patients initially diagnosed with various grades and types of gliomas.

\section{Future perspective}

Bev is commonly used for treatment of recurrent glioma patients, but most patients demonstrate progressive disease after Bev initiation. Interestingly, a significant fraction of our long-term survivors showed progression on Bev with eventual stability. Therefore, it remains important to continue identifying long-term survivors who benefit from Bev treatment and correlate these outcomes with molecular, clinical and imaging biomarkers. As our experience with these patients accumulates, we hope to be able to prospectively identify long-term survivors as well as understand imaging and clinical changes that occur during treatment so that Bev therapy is not prematurely discontinued for such patients. In addition, further understanding is necessary to determine optimal duration of Bev treatment for long-term survivors and to track chronic side effects of long-term Bev treatment. We believe that further studies such as ours will contribute to more optimal utilization of Bev for recurrent glioma patients.

\section{Executive summary}

- Long-term surviving recurrent glioma patients on Bev therapy are rarely reported in the current literature.

- We characterized a group of 50 long-term surviving recurrent glioma patients (Bev-LTS): 28 grade-IV, 14 grade III, and 8 grade II glioma patients who survived 3 years or greater after initiating Bev treatment.

- 45 of these 50 recurred on initial Bev treatment and of those, 36 patients were continued on Bev despite recurrence, which may suggest that long-term survival is still possible after initial failure of Bev treatment.

- Bev remains as one of the most widely used therapies available for recurrent glioma and continuing to identify characteristics of these long-term surviving patients remains important.

Supplementary data

To view the supplementary data that accompany this paper please visit the journal website at: www.futuremedicine.com/doi/full/ $10.2217 /$ cns-2019-0007

Acknowledgments

The authors thank Siliconmed for data aggregation.

Financial \& competing interests disclosure

Funding from UCLA SPORE in BrainCancer (P50 CA211015) to A Lai, TF Cloughesy, BM Ellingson, PL Nghiemphu and LM Liau and Art of the Brain (710 Westwood Plaza, Reed BLDG RM 1-230, CA 90095, USA) to A Lai; Bradley Zankel Foundation to A Lai. TF Cloughesy has received personal fees from Pfizer (NY, USA), Tocagen (CA 92121, USA), Roche (IN, USA), Novocure (NH, USA), Nektar (CA, USA), VBL (St.Modi'in, Israel), AbbVie (CA, USA), Upshire SmithPI (MN, USA), Notable Labs (CA, USA), Oxigene (CA 94080, USA), NewGen (TX, USA), Agios (MA, USA), Cortice (NY, USA), MedQia (CA, USA), PRoNai (BC V6C 3E8, Canada), Wellcome (London, NW1 2BE, UK), Merck (NJ, USA), Insys (AZ, USA), Human Longevity (CA 92121, USA), Sunovion (MA, 
USA), Boston Biomedical (MA, USA), Alexion (MA, USA), and Novogen (Pledran, France) outside the submitted work. TF Cloughesy is a board member of the Global Coalition for Adaptive Research 501c3 and the Principle Investigator for GBM Agile. TF Cloughesy has stock options for Notable Labs. A Lai has received honoraria from Merck, Genentech (CA, USA), AbbVie and Optune (NY, USA). PL Nghiemphu has received grants from Genentech/Roche. The authors have no other relevant affiliations or financial involvement with any organization or entity with a financial interest in or financial conflict with the subject matter or materials discussed in the manuscript apart from those disclosed.

No writing assistance was utilized in the production of this manuscript.

Ethical conduct of research

The authors state that they have obtained appropriate institutional review board approval or have followed the principles outlined in the Declaration of Helsinki for all human or animal experimental investigations. In addition, for investigations involving human subjects, informed consent has been obtained from the participants involved.

Informed consent disclosure

The authors state that they have obtained verbal and written informed consent from the patient/patients for the inclusion of their medical and treatment history within this case report.

\section{Open access}

This work is licensed under the Attribution-NonCommercial-NoDerivatives 4.0 Unported License. To view a copy of this license, visit http://creativecommons.org/licenses/by-nc-nd/4.0/

\section{References}

Papers of special note have been highlighted as: • of interest; $\bullet \bullet$ of considerable interest.

1. Bates DO. Vascular endothelial growth factors and vascular permeability. Cardiovasc Res. 87(2), 262-271 (2010).

2. Gerstner ER, Duda DG, di Tomaso E et al. VEGF inhibitors in the treatment of cerebral edema in patients with brain cancer. Nat. Rev. Clin. Oncol. 6, 229-236 (2009).

3. Yang SB, Gao KD, Jiang T et al. Bevacizumab combined with chemotherapy for glioblastoma: a meta-analysis of randomized controlled trials. Oncotarget. 8, 57337-57344 (2017).

4. Wick W, Gorlia T, Bendszus M et al. Lomustine and bevacizumab in progressive glioblastoma. N. Engl. J. Med. 377, 1954-1963 (2017).

-. A Phase III trial on the efficacy of bevacizumab in recurrent glioblastoma patients.

5. Gramatzki D, Roth P, Rushing EJ et al. Bevacizumab may improve quality of life, but not overall survival in glioblastoma: an epidemiological study. Ann. Oncol. 29(6), 1436 (2018).

6. Friedman HS, Prados MD, Wen PY et al. Bevacizumab alone and in combination with irinotecan in recurrent glioblastoma. J. Clin. Oncol. 27(28), 4733-4740 (2009).

7. Weathers SP, Han X, Liu DD et al. A randomized Phase II trial of standard dose bevacizumab versus low dose bevacizumab plus lomustine (CCNU) in adults with recurrent glioblastoma. J. Neurooncol. 129(3), 487-494 (2016).

8. Taal W, Oosterkamp HM, Walenkamp AM et al. Single-agent bevacizumab or lomustine versus a combination of bevacizumab plus lomustine in patients with recurrent glioblastoma (BELOB trial): a randomised controlled Phase II trial. Lancet Oncol. 15(9), 943-953 (2014).

9. Piccioni DE, Selfridge J, Mody RR et al. Deferred use of bevacizumab for recurrent glioblastoma is not associated with diminished efficacy. Neuro Oncol. 16(6), 815-822 (2014).

- Shows that patients may be able to defer bevacizumab use without diminishing overall survival time.

10. Iwamoto FM, Abrey LE, Beal $\mathrm{K}$ et al. Patterns of relapse and prognosis after bevacizumab failure in recurrent glioblastoma. Neurology 73(15), 1200-1206 (2009).

11. van den Bent MJ, Klein M, Smits M et al. Bevacizumab and temozolomide in patients with first recurrence of WHO grade II and III glioma, without 1p/19q co-deletion (TAVAREC): a randomised controlled Phase II EORTC trial. Lancet Oncol. 19(9), 1170-1179 (2018).

-• A randomized Phase II trial analyzing the effect of bevacizumab treatment on overall survival in recurrent grade II and -III glioma patients.

12. Ostrom QT, Gittleman H, Xu J et al. CBTRUS statistical report: primary brain and other central nervous system tumors diagnosed in the United States in 2009-2013. Neuro Oncol. 18(5), 1-75 (2016).

13. Michaelsen SR, Urup T, Olsen LR et al. Molecular profiling of short-term and long-term surviving patients identifies CD34 mRNA level as prognostic for glioblastoma survival. J. Neurooncol. 137(3), 533-542 (2018). 
14. Schomas DA, Laack NNI, Rao RD et al. Intracranial low-grade gliomas in adults: 30-year experience with long-term follow-up at Mayo Clinic. Neuro Oncol. 11(4), 437-445 (2009).

15. Spitaels J, Devriendt D, Sadeghi N et al. Management of supratentorial recurrent low-grade glioma: a multidisciplinary experience in 35 adult patients. Oncol. Lett. 14(3), 2789-2795 (2017).

16. Combs SE, Ahmadi R, Schulz-Ertner D et al. Recurrent low-grade gliomas: the role of fractionated stereotactic re-irradiation. J. Neurooncol. 71(3), 319-323 (2005).

17. Nahed BV, Redjal N, Brat DJ et al. Management of patients with recurrence of diffuse low grade glioma. J. Neurooncol. 125(3), 609-630 (2015).

18. Schweneker K, Clemm C, Brügel M et al. Effective long-term treatment with bevacizumab for relapsed glioblastoma: case report and review of the literature. Exp. Hematol. Oncol. 3, 29 (2018).

19. Vivekanandarajah A, Krishnarasa B, Mourad M et al. Achievement of three year remission with bevacizumab and irinotecan in recurrent glioblastoma multiforme: a case report. Clin. Med. Insights Oncol. 5, 15-21 (2011).

20. Balaña C, Estival A, Pineda E et al. Prolonged survival after bevacizumab rechallenge in glioblastoma patients with previous response to bevacizumab. Neurooncol. Prac. 4(1), 15-23 (2017).

21. van den Bent MJ, Weller M, Wen PY et al. A clinical perspective on the 2016 WHO brain tumor classification and routine molecular diagnostics. Neuro Oncol. 19(5), 614-624 (2017).

22. Dempsey MF, Condon BR, Hadley DM. Measurement of tumor 'size' in recurrent malignant glioma: 1D, 2D, or 3D? AJNR Am. J. Neuroradiol. 26(4), 770-776 (2005).

23. Nguyen HT, Nguyen N, Liu LY et al. Bevacizumab at first recurrence after standard radio-chemotherapy is associated with improved overall survival in glioblastoma patients with large tumor burden. Neurooncol. Prac. 6(2), 103-111 (2019).

- Suggests that tumor burden before bevacizumab initiation can have prognostic value in glioblastoma patients.

24. Reardon DA, Herndon JE, Peters KB et al. Bevacizumab continuation beyond initial bevacizumab progression among recurrent glioblastoma patients. Br. J. Cancer 107, 1481-1487 (2012).

-• Suggests that treatment continuation beyond initial recurrence on treatment may provide modest survival improvement in recurrent glioblastoma patients when compared with other nonbevacizumab therapies.

25. Tabouret $\mathrm{E}$, Barrie $\mathrm{M}$, Thiebaut $\mathrm{A}$ et al. Limited impact of prognostic factors in patients with recurrent glioblastoma multiforme treated with a bevacizumab-based regimen. J. Neurooncol. 114(2), 191-198 (2013).

26. Urup T, Dahlrot RH, Grunnet K et al. Development and validation of a prognostic model for recurrent glioblastoma patients treated with bevacizumab and irinotecan. Acta Oncol. 55(4), 418-422 (2016).

27. Krex D, Klink B, Hartmann C et al. Long-term survival with glioblastoma multiforme. Brain 130(10), 2596-2606 (2007).

28. Hamza MA, Mandel JJ, Conrad CA et al. Survival outcome of early versus delayed bevacizumab treatment in patients with recurrent glioblastoma. J. Neurooncol. 119(1), 135-140 (2014).

29. De Groot JF, Fuller G, Kumar AJ et al. Tumor invasion after treatment of glioblastoma with bevacizumab: radiographic and pathologic correlation in humans and mice. Neuro Oncol. 12(3), 233-242 (2010).

30. Gerber NK, Goenka A, Turcan S et al. Transcriptional diversity of long-term glioblastoma survivors. Neuro Oncol. 16(9), 1186-1195 (2014).

- Interestingly shows that $I D H$ mutation does not seem to impart long-term survival to glioblastoma patients ( $>4$ years) even though it is important in terms of short-term survival. MGMT methylation status is also shown to have prognostic value beyond 4 years.

31. Cantero D, de Lope AR, de la Presa RM et al. Molecular study of long-term survivors of glioblastoma by gene-targeted next-generation sequencing. J. Neuropathol. Exp. Neurol. 77(8), 710-716 (2018).

- Highlights genetic alterations, such as $A T R X$ and PDGFRA, that may have prognostic value for long-term surviving glioblastoma patients. 